\section{Higiene de las manos: conocimientos de los profesionales y áreas de mejora}

\author{
Hand hygiene: health professionals' knowledge \\ and areas for improvement
}

\author{
A higiene das mãos: as competências profissionais \\ e as áreas de melhoria
}

\author{
1 Observatorio para la \\ Seguridad del Paciente, \\ Agencia de Calidad Sanitaria \\ de Andalucía, Sevilla, \\ España. \\ 2 Agencia de Calidad \\ Sanitaria de Andalucía, \\ Sevilla, España. \\ 3 Departamento de \\ Sociología, Universidad de \\ Sevilla, Sevilla, España. \\ 4 Facultad de Medicina, \\ Universidad de Granada, \\ Granada, España. \\ 5 Distrito de Atención \\ Primaria Almería, Almería \\ España. \\ Correspondencia \\ M. Herrera-Usagre \\ Agencia de Calidad Sanitaria \\ de Andalucía. \\ C. Augusto Peyré s/n, Edif \\ Olalla, 3a Planta, Sevilla \\ 41020, España. \\ manuel.herrera.usagre@ \\ juntadeandalucia.es
}

\begin{abstract}
The objective of this study was to analyze knowledge on hand hygiene among staff workers in the Andalusian Public Health System (Spain). This was a cross-sectional study with surveys (2011) using the Hand Hygiene Knowledge Assessment Questionnaire for Healthcare Workers with the latent class analysis technique. The average number

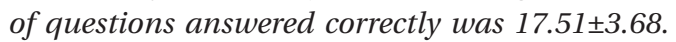
Questions with lower percentages of correct responses were those on hand hygiene for prevention of microorganism transmission to patients and those on hand-rubbing versus hand-washing. We obtained a model with 7 latent classes. Workers with lower knowledge tended to be younger, males, and non-healthcare workers. Having received previous training did not necessarily ensure excellent knowledge. The study concludes that hand hygiene training programs need to be revised in order to improve knowledge on conceptual characteristics involved in the transmission of microorganisms via the hands.
\end{abstract}

Hand Hygiene; Cross Infection; Patient Safety; Health Knowledge, Attitudes, Practice
Pastora Pérez-Pérez 1

Manuel Herrera-Usagre 2,3

Aurora Bueno-Cavanillas 4

María Soledad Alonso-Humada 5

Begoña Buiza-Camacho 2

Marta Vázquez-Vázquez ${ }^{1}$

\section{Resumen}

El objetivo fue analizar los conocimientos sobre la higiene de las manos de los profesionales del Sistema Sanitario Público Andaluz (España). Estudio mediante encuestas en 2011, utilizando el Hand Hygiene Knowledge Questionnaire for HealthCare Workers con la técnica de análisis de clases latentes. La media de respuestas correctas fue de $17,51 \pm 3,68$. Las preguntas con porcentajes de respuestas correctas más bajos son las referidas a la prevención, mediante la higiene de las manos, de la transmisión de microorganismos después de la exposición al paciente y aquellas relacionadas con la utilización de preparados de base alcohólica. Se obtuvo un modelo de 7 clases latentes. Principales diferencias socio-demográficas: los profesionales con conocimientos más bajos suelen ser hombres, jóvenes y no sanitarios; el haber recibido formación previa no discrimina a los que presentan excelentes conocimientos. Concluimos que la revisión de los programas de formación en higiene de las manos es necesaria para mejorar los conocimientos sobre aspectos conceptuales de la transmisión de microorganismos a través de las manos.

Higiene de las Manos; Infección Hospitalaria; Seguridad del Paciente; Conocimientos, Actitudes y Práctica en Salud 


\section{Introducción}

Las infecciones relacionadas con la asistencia sanitaria son las que adquieren los pacientes derivadas de la asistencia sanitaria y que no estaban presentes o incubándose en el momento de recibirla. Estas infecciones constituyen un grave problema de salud, ya que se encuentran entre las principales causas de mortalidad y de incremento de la morbilidad en los pacientes, especialmente en los países en vías de desarrollo ${ }^{1}$.

Según la Organización Mundial de la Salud (OMS), la prevalencia de infecciones relacionadas con la asistencia sanitaria en países desarrollados se sitúa en 7,6 infecciones por cada 100 pacientes (IC95\%: 6,9-8,5) 1, mientras que en los países en desarrollo es de 15,5 por cada 100 pacientes (IC95\%: 12,6-18,9) 2. En Europa, según datos del European Centre for Disease Prevention and Control, la prevalencia se sitúa en un $7,1 \%$ de media con un rango entre el $3,5 \%$ y el $10,5 \% 3$. Esto se traduce en 4 millones de pacientes infectados y 40.000 muertes directas atribuibles a las infecciones relacionadas con la asistencia sanitaria, sólo en Europa 1. En España, según el último informe Estudio de Prevalencia de las Infecciones Nosocomiales en España (EPINE) ${ }^{4}$ para los hospitales, las infecciones nosocomiales poseen una prevalencia que se sitúa en la media europea $(7,1 \%)$, con un rango que va del $6,9 \%$ al $7,3 \%$. Por otro lado, en el ámbito de la atención primaria de salud, el 7,4\% de los eventos adversos que se producen en los centros de salud españoles está relacionado con las infecciones relacionadas con la asistencia sanitaria 5 .

Ante la relevancia del problema y el riesgo significativo para la seguridad de los pacientes en todos los países, en el año 2005, la OMS proyectó a nivel mundial el reto "una atención limpia es una atención más segura” (clean care is safer care), haciendo clara referencia a las infecciones relacionadas con la asistencia sanitaria y a sus estrategias de prevención 6 . Entre las acciones clave, la higiene de las manos emerge como la medida más sencilla, barata y eficaz para evitar las infecciones 6,7,8. Con el fin de promover esta acción a cualquier escala (estatal, regional o local), el programa se amplió en el 2009 centrándose en "los 5 momentos para la higiene de las manos” de la atención sanitaria 9,10. Al mismo tiempo, se elaboró una guía con una batería de actuaciones, aplicable a cualquier centro sanitario, que permitiese llevar a la práctica las directrices de la OMS para la higiene de las manos y mejorar su cumplimiento 11 , ampliándose estas recomendaciones posteriormente a otros ámbitos 12. Entre las actuaciones que se recomiendan, surgen como componentes esenciales, la forma- ción periódica sobre la importancia de la higiene de las manos y los procedimientos adecuados para la fricción y el lavado de las manos, así como la evaluación regular de las infraestructuras, prácticas, conocimientos y percepciones de los profesionales 11,12.

Numerosos estudios han venido demostrando hasta ahora la importancia de la medición de los conocimientos, los riesgos, las actitudes y las percepciones de los profesionales hacia la higiene de las manos, como medio para el diseño de programas de prevención de infecciones relacionadas con la asistencia sanitaria en cualquier nivel asistencial 13,14,15,16,17,18. En este sentido, una adecuada formación tanto en las etapas pregrado 19 , como en la etapa profesional 20 , a través de intervenciones formativas específicas, se presenta igualmente como un medio de intervención eficaz. De hecho, muchos de estos estudios han evidenciado el impacto positivo de la formación en higiene de las manos, tanto en los conocimientos y las percepciones de los profesionales sanitarios 14,17,21, como en su adecuación a los estándares y prácticas seguras 17,22,23.

A pesar de la discusión científica existente sobre las mejores estrategias de intervención para la mejora de la higiene de las manos, la inmensa mayoría de los estudios coinciden en la necesidad de realizar una primera fase de diagnóstico sobre los conocimientos de los profesionales y así implementar lo que se conocen como stateof-the-art strategies o estrategias que atacan al estado de la cuestión, es decir, formación, recordatorios, facilidades y productos 24,25 . Han sido varios los cuestionarios utilizados para evaluar los conocimientos sobre infecciones relacionadas con la asistencia sanitaria y la higiene de las manos 26,27,28, hasta que la OMS incluyó dentro de su guía de aplicación el Hand Hygiene Knowledge Questionnaire for Health-Care Workers ${ }^{11}$.

El objetivo del presente trabajo es analizar los conocimientos sobre la higiene de las manos demostrados por los profesionales del Sistema Sanitario Público de Andalucía, España, mediante este cuestionario, con vistas a la identificación, priorización y puesta en marcha de acciones de mejora. Igualmente, se evaluará el impacto de la formación recibida en higiene de las manos y las posibles diferencias de conocimientos, en base a las características individuales de los profesionales. 


\section{Material y método}

\section{Diseño}

Estudio analítico en forma de encuestas trasversales.

\section{Ámbito de estudio}

El Sistema Sanitario Público de Andalucía es una organización integrada por 102.000 profesionales, que dispone de una red de centros asistenciales para garantizar la continuidad en la atención sanitaria. Concretamente, la atención primaria de salud se presta desde 1.514 centros sanitarios (centros de salud, consultorios locales y auxiliares) y 489 dispositivos de cuidados críticos y urgencias, y la atención hospitalaria se realiza desde 83 centros, de los que 48 son hospitales públicos y, el resto, centros de consultas externas especializadas 29 .

\section{Sujetos de estudio}

Profesionales del Sistema Sanitario Público Andalucía que de forma voluntaria han cumplimentado el cuestionario online, a través de la página web del Observatorio para la Seguridad del Paciente, Agencia de Calidad Sanitaria de Andalucía 30.

Dado que la cumplimentación de las encuestas fue voluntaria, se tuvo que comprobar si la distribución porcentual de categorías profesionales de la muestra (enfermeras, médicos y resto de profesionales de la salud que trabajan en el sistema) no variaba de manera estadísticamente significativa del universo de referencia, a saber, la población de profesionales del Sistema Sanitario Público Andalucía. Una vez comprobado, podemos considerar la muestra como representativa 31 .

El estudio fue realizado en el año de 2011.

\section{Herramienta}

Fue utilizado el Cuestionario de Conocimientos sobre la Higiene de las Manos para Profesionales Sanitarios (Hand Hygiene Knowledge Questionnaire for Health-Care Workers, traducido por el Ministerio de Sanidad, Política Social e Igualdad y validado por la OMS) 32 . El cuestionario incluye 25 preguntas sobre los aspectos esenciales de la higiene de las manos y la transmisión de microorganismos a través de las manos durante la atención sanitaria. Además, contiene preguntas con el objeto de describir la población de estudio y posibilitar la comparación entre distintos subgrupos: sexo, edad, categoría profesional y ámbito de trabajo, más dos preguntas sobre la formación recibida en higiene de las manos y la utilización de preparados de base alcohólica de forma habitual. Las categorías profesionales se distribuyen entre: (1) enfermeras, que incluyen enfermeras y matronas; (2) médicos, que incluyen médicos y residentes; (3) otros profesionales de la salud, que incluyen auxiliares de enfermería, técnicos (radiología, cardiología, laboratorio, etc.), terapeutas (psicoterapeutas, terapeutas ocupacionales, audiólogos, logopedas, etc.), estudiantes de enfermería, de medicina, y resto de profesionales no mencionados anteriormente (dietistas, dentistas, trabajadores sociales, farmacéuticos, etc.).

\section{Aspectos éticos}

La información aportada a los profesionales resalta el hecho del carácter voluntario y anónimo de las encuestas, así como del cumplimiento de la normativa vigente en España (Ley n. 14/2007 de Investigación Biomédica, Ley Orgánica $n$. 15/1999 de Protección de Datos de Carácter Personal y la Ley $n$. 41/2002 Básica Reguladora de la Autonomía del Paciente y de Derechos y Obligaciones en Materia de Información y Documentación Clínica), asegurando en todo momento la confidencialidad y anonimidad de los datos obtenidos. Además, el Comité Coordinador de ética de la investigación biomédica de Andalucía considera que el estudio se ajusta a los principios éticos aplicables.

\section{Procesamiento y análisis de datos}

Todas las respuestas relacionadas con la higiene de las manos y la transmisión de microorganismos se han codificado en 1 "respuesta correcta" y 0 "respuesta incorrecta/no sabe, no contesta". La descripción de las variables cualitativas categóricas se muestra en forma de distribución de frecuencias. Como técnica analítica se ha realizado un análisis de clases latentes o latent class analysis (LCA) para extraer una serie de grupos o clases latentes de profesionales, en función de sus conocimientos demostrados. La introducción de covariables en el modelo permitirá, adicionalmente, predecir las diferentes características de los profesionales dentro de cada clase latente 33 . Básicamente, el LCA es una técnica que permite la clasificación de los individuos en diferentes clases o clusters, a través de una aproximación probabilística. A pesar de sus semejanzas con otros tipos de análisis de clasificación, considerados de poca robustez o fuzzy clustering, el LCA presenta una serie de características propias que han propiciado su mayor utilización en los 
últimos años. Según Vermunt \& Magidson 34, una diferencia importante es que, si bien en otras técnicas de fuzzy clustering, el grado de pertenencia de un objeto (a una clase) son los "parámetros" que han de ser estimados; en el LCA, las probabilidades que tiene un individuo de pertenecer a una clase son directamente computadas de los parámetros del modelo estimado y sus puntuaciones observadas. En otras palabras, el LCA permite clasificar otros objetos pertenecientes a la población de la que se ha tomado la muestra, lo cual no es posible a través de otras técnicas de fuzzy clustering estándar 33 . Para la realización del modelo de clases latentes hemos utilizado el paquete poLCA del software R package (The R Foundation for Statistical Computing, Viena, Austria; http://www.r-project.org) 35.

En el modelo se incluyen todas las preguntas del test, excepto aquellas que obtengan una tasa de respuesta correcta superior al 90\%. Esto asegura incluir solo aquellas preguntas que dada su gran variabilidad tienen una gran capacidad para discriminar a los individuos en función de sus conocimientos. De este modo, se podrá comprobar qué características de los profesionales están asociadas a un mayor desconocimiento en según qué aspectos, haciendo más eficaces las diferentes estrategias multimodales de mejora de la higiene de las manos.

Se recibieron 2.448 cuestionarios, siendo la muestra analítica final de 1.837 casos, una vez eliminados aquellos que habían dejado alguna pregunta socio-demográfica sin responder.

Antes de profundizar en los resultados del análisis de clases latentes, se ha de identificar aquel modelo con el número de clases más parsimonioso. Para ello, se compararon los modelos utilizando dos criterios de información estadística, AIC (Akaike Information Criterion) y BIC (Bayesian Information Criterion). Ambos criterios permiten comprobar qué modelo se ajusta más a la realidad. Mientras que el AIC está optimizado para averiguar el mejor modelo en un número infinito de modelos, el BIC lo está para averiguar el mejor modelo de un número finito de ellos. El modelo a escoger tiende a ser aquel que tenga un BIC inferior. Se analizaron modelos de 2 a 20 clases latentes. El modelo final escogido fue el de 7 clases, dado que a partir de ese número no hubo una reducción sustancial del estadístico BIC.

\section{Resultados}

\section{Análisis descriptivo}

La edad media de los profesionales que lo cumplimentaron fue de 45,45 \pm 8,92 años. La descrip- ción de la población se presenta en la Tabla 1. En cuanto a las preguntas del cuestionario, la media de preguntas contestadas correctamente fue de $17,51 \pm 3,61$. La frecuencia y porcentaje de respuestas correctas por pregunta se muestran en la Tabla 2.

Las preguntas con porcentajes de respuestas correctas más bajos son las referidas a la prevención, mediante la higiene de las manos, de la transmisión de microorganismos a los pacientes después de la exposición (preguntas P15b y P15c) o al personal sanitario antes de la exposición (P16a y P16d). Por otra parte, hay que destacar que sólo un $40 \%$ de los profesionales afirma correctamente que tras el lavado de las manos con agua y jabón no es necesario realizar fricción con preparados de base alcohólica (P17d).

En el otro extremo, se sitúan las preguntas con unos porcentajes de respuestas correctas superiores al $90 \%$. Estas preguntas están relacionadas con la identificación de la vía principal para la transmisión cruzada de microorganismos (P13), con la prevención mediante la higiene de las manos de la transmisión de microorganismos a los pacientes antes de la exposición (P15a y P15d), o al personal sanitario después de la exposición (P16b y P16c), y con otras actuaciones de prevención de infecciones no relacionadas con la higiene de las manos (P20a, P20b y P20c).

\section{Identificación de tipologías de profesionales en cuanto a sus conocimientos}

Las características más destacadas a nivel general de cada una de las 7 clases latentes, en cuanto a los conocimientos sobre la higiene de las manos y la prevención de infecciones relacionadas con la asistencia sanitaria, se presentan en la Tabla 3.

La Figura 1 ilustra la estructura de interacciones entre las preguntas del cuestionario en cada una de las siete clases latentes. El eje X muestra la totalidad de preguntas incluidas en el LCA, mientras que el eje Y muestra el porcentaje de probabilidad para los individuos clasificados en cada clase latente de que contesten correctamente a cada pregunta. La Figura 1 se interpreta del siguiente modo: los profesionales incluidos en la clase Excelente acaparan el 11,91\% de los casos, y tienen un $93,75 \%$ de probabilidades de responder correctamente a la pregunta p14; un $100 \%$ de probabilidades de responder correctamente a la pregunta P15c y un $69,82 \%$ de responder bien a la pregunta P17d. Por otro lado los profesionales de la clase Deficitaria agrupan a un $9,13 \%$ de los casos y tienen un $42,9 \%$ de probabilidades de responder correctamente a la pregunta p14; un 4,06\% de probabilidades de 
Descripción de la población de estudio ( $\mathrm{N}=1.837)$.

\begin{tabular}{|c|c|c|}
\hline Variables & $\mathrm{n}$ & $\%$ \\
\hline \multicolumn{3}{|l|}{ Ámbito de trabajo } \\
\hline Atención primaria & 914 & 49,8 \\
\hline Atención hospitalaria & 923 & 50,2 \\
\hline \multicolumn{3}{|l|}{ Sexo } \\
\hline Mujer & 1.304 & 71,0 \\
\hline Hombre & 533 & 29,0 \\
\hline \multicolumn{3}{|l|}{ Categoría profesional } \\
\hline Médicos & 830 & 45,2 \\
\hline Enfermeras & 473 & 25,7 \\
\hline Otros profesionales de la salud & 534 & 29,1 \\
\hline \multicolumn{3}{|l|}{ Edad (años) } \\
\hline$\leq 35$ & 285 & 15,5 \\
\hline $36-45$ & 592 & 32,2 \\
\hline $46-55$ & 739 & 40,2 \\
\hline$>55$ & 221 & 12,1 \\
\hline \multicolumn{3}{|c|}{ Formación previa en higiene de las manos en los últimos 3 años } \\
\hline Sí & 1.497 & 81,5 \\
\hline No & 340 & 18,5 \\
\hline \multicolumn{3}{|c|}{ Utilización de preparados de base alcohólica de forma habitual } \\
\hline Sí & 1.636 & 89,1 \\
\hline No & 201 & 10,9 \\
\hline Total & 1.837 & 100,0 \\
\hline
\end{tabular}

responder correctamente a la pregunta P15c y un $26,86 \%$ de responder bien a la pregunta $\mathrm{P} 17 \mathrm{~d}$.

Para comprobar qué características definen mejor a cada clase, se realizó una serie de regresiones logísticas multinomiales. La Tabla 4 compara las características de todos los grupos, frente a la clase con mayor tasa de respuestas correctas, la clase Excelente.

De los resultados de la Tabla 4 destaca el hecho, en términos generales, de que no existen grandes diferencias entre la clase con mayor nivel de conocimientos (clase Excelente) con las clases Avanzada, Avanzada en técnicas, Déficit en transmisión y Déficit en adecuación, aunque sí existen con las clases Déficit en conceptos y Deficitaria. No obstante, caben señalar algunas diferencias. La clase excelente se caracteriza por una alta presencia de mujeres si la comparamos con el resto de clases. Además, esta clase contiene un mayor número de enfermeras y médicos que la clase déficit en conceptos y que la clase deficitaria, donde hay una mayor presencia de otros profesionales de la salud. Los profesionales de la clase excelente proceden de manera más o menos equitativa del ámbito hospitalario y de la atención primaria, aunque su presencia es más común en el ámbito de primaria que la clase Déficit en conceptos, y más común en el ámbito hospitalario que la clase Deficitaria. La edad tampoco parece diferenciar a aquellos que tienen los niveles más altos de conocimientos, a excepción de la clase Deficitaria, que se diferencia por ser más joven que la clase Excelente. Es preciso destacar que el haber recibido o no formación no discrimina a los que presentan excelentes conocimientos sobre la higiene de las manos (clase Excelente) del resto. Por último, la clase de profesionales que tiene un mayor déficit en conceptos sobre la transmisión de patógenos al paciente y al profesional, la clase Déficit en transmisión, tiene más probabilidades de declarar que habitualmente usa preparados de base alcohólica que la clase Excelente.

\section{Discusión}

El objetivo del presente estudio es analizar los conocimientos sobre higiene de las manos, demostrados por los profesionales del Sistema Sanitario Público de Andalucía, con vistas a la 
Tabla 2

Frecuencia y porcentaje de respuestas correctas por pregunta $(N=1.837)$.

\begin{tabular}{|c|c|c|c|}
\hline $\begin{array}{l}\text { Tipología de } \\
\text { preguntas }\end{array}$ & Preguntas (respuesta correcta) & n & $\%$ \\
\hline \multicolumn{4}{|l|}{ Conceptos } \\
\hline Vía & $\begin{array}{l}\text { P13. ¿Cuál de las siguientes es la vía principal para la transmisión cruzada de microorganismos } \\
\text { potencialmente patógenos en una institución sanitaria? (manos de los trabajadores) }\end{array}$ & 1.748 & 95,2 \\
\hline Fuente & $\begin{array}{l}\text { P14. ¿Cuál es la fuente de infección más frecuente para los microorganismos responsables de las } \\
\text { infecciones asociadas a la asistencia sanitaria? (microorganismos que colonizan al paciente) }\end{array}$ & 1.010 & 55,0 \\
\hline \multirow[t]{5}{*}{$\begin{array}{l}\text { Transmisión al } \\
\text { paciente }\end{array}$} & $\begin{array}{l}\text { P.15 ¿En cuál de las siguientes ocasiones la higiene de manos previene la transmisión de } \\
\text { microorganismos al paciente? }\end{array}$ & & \\
\hline & a. Antes de tocar al paciente (sí) & 1.734 & 94,4 \\
\hline & b. Inmediatamente después de la exposición a fluidos corporales (no) & 406 & 22,1 \\
\hline & c. Después de la exposición al entorno del paciente (no) & 616 & 33,5 \\
\hline & d. Inmediatamente antes de realizar un procedimiento aséptico (sí) & 1.652 & 89,9 \\
\hline \multirow[t]{5}{*}{$\begin{array}{l}\text { Transmisión al } \\
\text { profesional }\end{array}$} & $\begin{array}{l}\text { P16. ¿En cuál de las siguientes ocasiones la higiene de manos previene la transmisión de } \\
\text { microorganismos al personal sanitario? }\end{array}$ & & \\
\hline & a. Antes de tocar al paciente (no) & 884 & 48,1 \\
\hline & b. Inmediatamente después de la exposición a fluidos corporales (sí) & 1.723 & 93,8 \\
\hline & c. Después de la exposición al entorno del paciente (sí) & 1.659 & 90,3 \\
\hline & d. Inmediatamente antes de realizar un procedimiento aséptico (no) & 797 & 43,4 \\
\hline \multicolumn{4}{|l|}{ Técnicas } \\
\hline \multirow[t]{5}{*}{ Productos } & $\begin{array}{l}\text { P17. Indique si las siguientes afirmaciones sobre la higiene de manos con preparados de base } \\
\text { alcohólica o lavado con agua y jabón son verdaderas o falsas }\end{array}$ & & \\
\hline & $\begin{array}{l}\text { a. La fricción con preparados de base alcohólica es más rápida para la higiene de manos que el } \\
\text { lavado con agua y jabón (verdadera) }\end{array}$ & 1.553 & 84,5 \\
\hline & $\begin{array}{l}\text { b. La fricción con solución alcohólica reseca las manos más intensamente que el lavado de manos } \\
\text { convencional (falso) }\end{array}$ & 1.006 & 54,8 \\
\hline & c. La fricción con solución alcohólica desinfecta más y mejor que el lavado de manos (verdadero) & 1.108 & 60,3 \\
\hline & $\begin{array}{l}\text { d. El lavado de manos y la fricción con solución alcohólica se deben realizar secuencialmente } \\
\text { (primero lavado y posteriormente fricción) (falso) }\end{array}$ & 734 & 40,0 \\
\hline Tiempo & $\begin{array}{l}\text { P18. ¿Cuál es el tiempo mínimo necesario para que la fricción con un preparado de base alcohólica } \\
\text { elimine la mayor parte de los microorganismos de sus manos? (20 segundos) }\end{array}$ & 1.305 & 71,0 \\
\hline \multirow[t]{7}{*}{ Adecuación } & P19. ¿Qué tipo de higiene de manos está indicada en las siguientes situaciones? & & \\
\hline & a. Antes de la palpación del abdomen (fricción) & 1.221 & 66,5 \\
\hline & b. Antes de poner una inyección (fricción) & 1.205 & 65,6 \\
\hline & c. Después de vaciar una cuña (lavado) & 1264 & 68,8 \\
\hline & d. Después de retirar unos guantes de exploración (fricción) & 969 & 52,7 \\
\hline & e. Después de hacer la cama del paciente (fricción) & 1.008 & 54,9 \\
\hline & f. Después de exposición visible a sangre (lavado) & 1.332 & 72,5 \\
\hline \multirow[t]{5}{*}{$\begin{array}{l}\text { Otros aspectos de } \\
\text { las técnicas }\end{array}$} & $\begin{array}{l}\text { P20. ¿Cuál de las siguientes situaciones debe evitarse, por su asociación con un aumento de la } \\
\text { probabilidad de colonización de las manos con microorganismos patógenos? }\end{array}$ & & \\
\hline & a. Llevar joyería (sí) & 1.764 & 96,0 \\
\hline & b. Piel lesionada (sí) & 1.741 & 94,8 \\
\hline & c. Uñas artificiales (sí) & 1.774 & 96,6 \\
\hline & d. Uso frecuente de crema de manos (no) & 1.164 & 63,4 \\
\hline
\end{tabular}


Tabla 3

Características esenciales de cada clase latente.

\begin{tabular}{|c|c|c|}
\hline Clase & Descripción breve & Descripción detallada \\
\hline Excelente & $\begin{array}{l}\text { Conocimientos excelentes } \\
\text { tanto en conceptos como en } \\
\text { técnicas }\end{array}$ & $\begin{array}{l}\text { Probabilidades muy altas (superiores al } 80 \% \text { ) de contestar } \\
\text { correctamente a todas las preguntas. }\end{array}$ \\
\hline Avanzada & $\begin{array}{l}\text { Conocimientos avanzados } \\
\text { tanto en conceptos como en } \\
\text { técnicas }\end{array}$ & $\begin{array}{l}\text { Probabilidades muy altas de contestar correctamente a las } \\
\text { preguntas relacionadas con las situaciones en las que la higiene de } \\
\text { las manos previene la transmisión de infecciones tanto al paciente } \\
\text { (P15), como al personal sanitario (P16), y unas probabilidades } \\
\text { moderadas de responder correctamente al resto. }\end{array}$ \\
\hline $\begin{array}{l}\text { Avanzada en } \\
\text { técnicas }\end{array}$ & $\begin{array}{l}\text { Conocimientos avanzados } \\
\text { en técnicas y deficitarios en } \\
\text { conceptos sobre transmisión } \\
\text { al paciente }\end{array}$ & $\begin{array}{l}\text { Probabilidades muy bajas (inferiores al } 20 \% \text { ) de contestar bien a las } \\
\text { preguntas relacionadas con las situaciones en las que la higiene de } \\
\text { las manos previene la transmisión de infecciones al paciente antes } \\
\text { de la exposición (P15) y muy altas en las referidas a la prevención } \\
\text { de infecciones al personal sanitario (P16). Con respecto a los } \\
\text { conocimientos sobre la higiene de las manos durante la atención } \\
\text { sanitaria (P17, P18 y P19), en general, las probabilidades de } \\
\text { contestar correctamente a las preguntas son altas (en torno al 60\%). }\end{array}$ \\
\hline $\begin{array}{l}\text { Déficit en } \\
\text { transmisión }\end{array}$ & $\begin{array}{l}\text { Conocimientos avanzados } \\
\text { en técnicas y deficitarios en } \\
\text { conceptos sobre transmisión } \\
\text { al paciente y al profesional }\end{array}$ & $\begin{array}{l}\text { Parecido a la clase Avanzada en técnicas, con la salvedad de que } \\
\text { tiene probabilidades muy inferiores de responder correctamente a } \\
\text { las preguntas referidas a los conocimientos sobre la transmisión de } \\
\text { infecciones a través de las manos al personal sanitario antes de la } \\
\text { exposición al paciente (P16a y P16d). }\end{array}$ \\
\hline $\begin{array}{l}\text { Déficit en } \\
\text { conceptos }\end{array}$ & $\begin{array}{l}\text { Conocimientos moderados } \\
\text { en técnicas y deficitarios en } \\
\text { conceptos. }\end{array}$ & $\begin{array}{l}\text { Probabilidades muy bajas (inferiores al 20\%) de responder } \\
\text { correctamente a las preguntas relacionadas con las situaciones } \\
\text { en las que la higiene de las manos previene la transmisión de } \\
\text { infecciones al paciente después de la exposición a este (P15b } \\
\text { y P15c) y bajas en las referidas al personal sanitario antes de la } \\
\text { exposición (P16a y P16d). Con respecto al resto de preguntas, } \\
\text { presentan una elevada dispersión en cuanto a su probabilidad de } \\
\text { respuesta }\end{array}$ \\
\hline $\begin{array}{l}\text { Déficit en } \\
\text { adecuación }\end{array}$ & $\begin{array}{l}\text { Conocimientos moderados } \\
\text { en conceptos y técnicas y } \\
\text { deficitarios en adecuación a } \\
\text { las técnicas. }\end{array}$ & $\begin{array}{l}\text { Probabilidades bajas (no superiores al 50\%) de contestar } \\
\text { correctamente a las preguntas relacionadas con las situaciones } \\
\text { en las que la higiene de las manos previene la transmisión de } \\
\text { infecciones en el paciente después de la exposición a este (P15b y } \\
\text { P15c). En cuanto a los conocimientos sobre la higiene de las manos } \\
\text { durante la atención sanitaria, tiene unas probabilidades muy bajas } \\
\text { (inferiores al 20\%) de contestar correctamente a las preguntas } \\
\text { relacionadas con el tipo de higiene de las manos más adecuado } \\
\text { ante situaciones concretas (P19). }\end{array}$ \\
\hline Deficitaria & $\begin{array}{l}\text { Conocimientos deficitarios en } \\
\text { conceptos y técnicas. }\end{array}$ & $\begin{array}{l}\text { Probabilidades muy bajas de contestar correctamente a las } \\
\text { preguntas relacionadas con las situaciones en las que la higiene de } \\
\text { las manos previene la transmisión de infecciones, tanto al paciente } \\
\text { antes de la exposición (P15b y P15c), como al personal sanitario } \\
\text { después de la exposición a aquel (P16a y P16d). No obstante, y } \\
\text { a diferencia de la clase 6, presenta conocimientos más sólidos } \\
\text { respecto a algunas de las preguntas sobre la higiene de las manos } \\
\text { durante la atención sanitaria (P19). }\end{array}$ \\
\hline
\end{tabular}


Figura 1

Probabilidades condicionales de asociación entre conocimientos demostrados en seguridad del paciente. Modelo de 7 clases latentes.

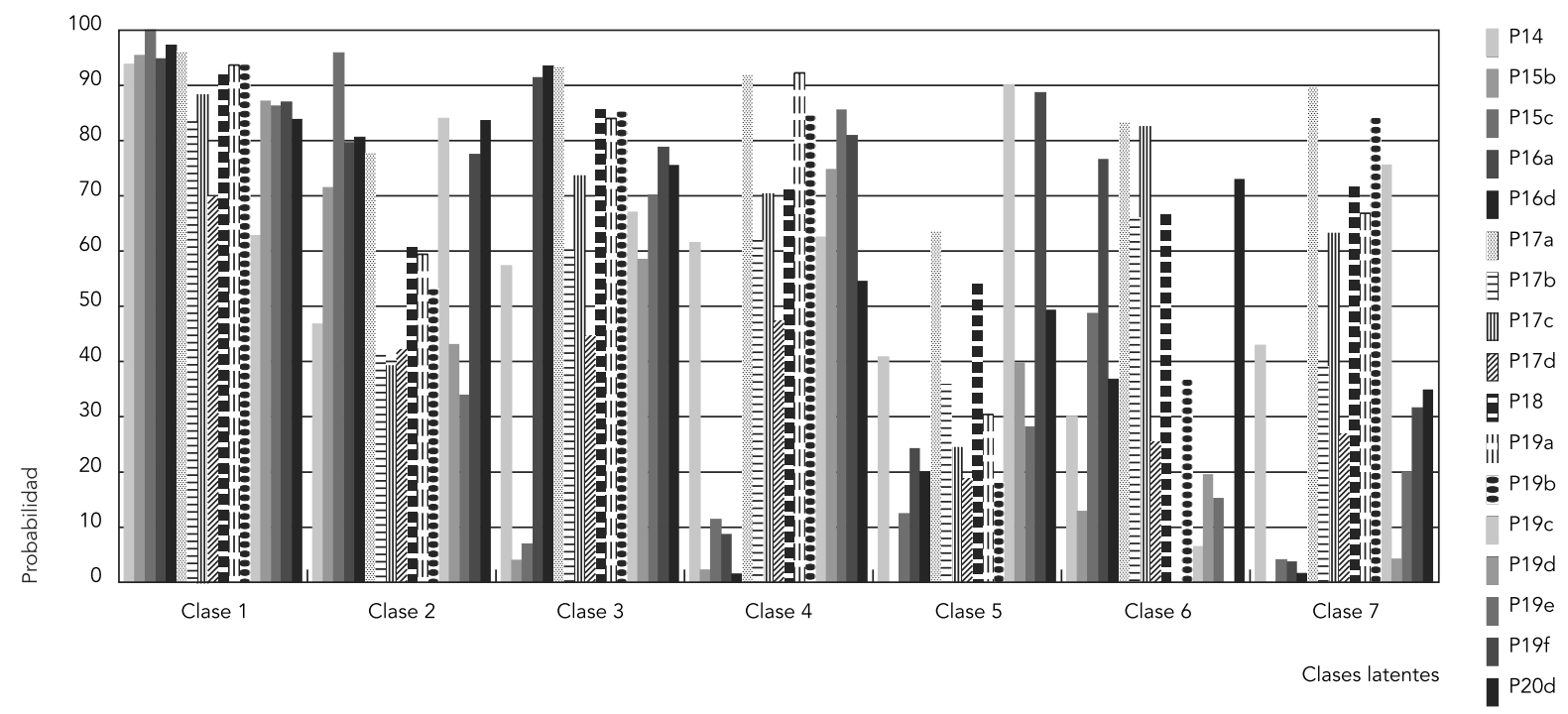

Clase 1: Excelente (11,91\%); Clase 2: Avanzada (12,19\%); Clase 3: Avanzada en técnicas (16,14\%); Clase 4: Déficit en trasmisión (24,26\%); Clase 5: Déficit en conceptos (19,83\%); Clase 6: Déficit en adecuación (6,63\%); Clase 7: Deficitaria (9,13\%).

identificación, priorización y puesta en marcha de acciones de mejora, así como evaluar el impacto de la formación recibida en higiene de las manos.

Los resultados aportados señalan la existencia de importantes lagunas de conocimiento en los profesionales. A nivel global, las mayores deficiencias de conocimiento se encuentran en aquellos aspectos relacionados con los conceptos de la higiene de las manos, por ejemplo: con las situaciones en las que la higiene de las manos previene la transmisión de microorganismos a pacientes o al personal sanitario (P15 y P16). También, existen importantes áreas de mejora en la formación sobre las técnicas, por ejemplo, sobre las ventajas o inconvenientes de utilizar preparados de base alcohólica frente al lavado con agua y jabón (P17).

Sin embargo, el presente estudio muestra la existencia de determinados perfiles profesionales en los que las diferencias de conocimientos están más acusadas. En primer lugar, las mujeres tienen una mayor probabilidad de tener mejores conocimientos en higiene de las manos que los hombres, aspecto que coincide con lo encontrado por Amin \& Al Wehedy 36 en su estudio. Por otro lado, si bien los resultados encontrados sugieren un menor conocimiento sobre higiene de las manos entre los profesionales de la salud, que no son ni médicos ni enfermeras, no se han encontrado diferencias entre el personal de enfermería y el médico. Estos resultados coincidirían así con los obtenidos por Bisset 37 , quien no encontró diferencias significativas sobre conocimientos de higiene de las manos entre enfermeras y médicos, pero sí con el resto de profesionales. Las deficiencias de conocimientos en este colectivo profesional tan heterogéneo se centran en las situaciones de riesgo de transmisión de infecciones para pacientes y profesionales (P15 y P16), así como en el tipo de higiene de las manos más adecuada ante según qué situaciones (P19). A pesar de que este grupo profesional, al no tener un contacto tan directo con los pacientes, no necesitaría poseer unos conocimientos tan exhaustivos sobre las situaciones de riesgo, su formación en la materia no debería ser minusvalorada.

Otro aspecto importante ha sido el impacto de la formación previa en higiene de las manos sobre los conocimientos adquiridos. Según los datos obtenidos, la formación no ha tenido todo el impacto esperado, a pesar de que otros estudios sí encuentren una relación positiva con la adhesión a la práctica 20,38 y han demostrado la eficacia de los programas formativos en la adherencia a prácticas de higiene de las ma- 
Análisis logístico multinomial sobre las características sociodemográficas de cada clase latente. Comparativa frente a la clase excelente.

\begin{tabular}{|c|c|c|c|c|c|c|c|c|c|c|c|c|}
\hline & \multicolumn{2}{|c|}{ Avanzada * } & \multicolumn{2}{|c|}{$\begin{array}{l}\text { Avanzada en } \\
\text { técnicas * }\end{array}$} & \multicolumn{2}{|c|}{$\begin{array}{l}\text { Déficit en } \\
\text { transmisión * }\end{array}$} & \multicolumn{2}{|c|}{$\begin{array}{l}\text { Déficit en } \\
\text { conceptos * }\end{array}$} & \multicolumn{2}{|c|}{$\begin{array}{c}\text { Déficit en } \\
\text { adecuación * }\end{array}$} & \multicolumn{2}{|c|}{ Deficitaria * } \\
\hline & $\begin{array}{l}\text { Coefi- } \\
\text { ciente }\end{array}$ & ES & $\begin{array}{l}\text { Coefi- } \\
\text { ciente }\end{array}$ & ES & $\begin{array}{l}\text { Coefi- } \\
\text { ciente }\end{array}$ & ES & $\begin{array}{l}\text { Coefi- } \\
\text { ciente }\end{array}$ & ES & $\begin{array}{l}\text { Coefi- } \\
\text { ciente }\end{array}$ & ES & $\begin{array}{l}\text { Coefi- } \\
\text { ciente }\end{array}$ & ES \\
\hline Constante & 0,691 & 0,789 & $-1,425$ & 0,812 & 0,023 & 0,678 & $-0,041$ & 0,687 & $-0,489$ & 0,914 & 1,201 & 0,865 \\
\hline Edad & 0,001 & 0,014 & 0,020 & 0,013 & 0,007 & 0,012 & 0,003 & 0,012 & $-0,022$ & 0,015 & $-0,035 * \star$ & 0,016 \\
\hline 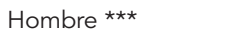 & 0,176 & 0,298 & $0,746 \#$ & 0,243 & 0,351 & 0,229 & 0,610 ** & 0,238 & 0,744 ** & 0,315 & 0,896 ** & 0,288 \\
\hline Médico \#\# & $-0,359$ & 0,321 & $-0,439$ & 0,258 & $-0,308$ & 0,230 & $-0,321$ & 0,256 & $-0,104$ & 0,367 & 0,055 & 0,334 \\
\hline $\begin{array}{l}\text { Otros } \\
\text { profesionales de la } \\
\text { salud \#\# }\end{array}$ & 0,376 & 0,289 & $-0,124$ & 0,268 & $-0,039$ & 0,242 & 0,481 ** & 0,242 & 0,661 & 0,316 & 0,861 \# & 0,329 \\
\hline $\begin{array}{l}\text { Atención } \\
\text { hospitalaria \#\#\# }\end{array}$ & $-0,107$ & 0,277 & $-0,290$ & 0,248 & $-0,196$ & 0,228 & 0,569 ** & 0,238 & $-0,281$ & 0,333 & $-0,650$ ** & 0,330 \\
\hline Formación previa & $-0,304$ & 0,336 & 0,401 & 0,337 & $-0,433$ & 0,274 & 0,114 & 0,277 & 0,185 & 0,427 & 0,274 & 0,419 \\
\hline $\begin{array}{l}\text { Usa de preparados } \\
\text { de base alcohólica }\end{array}$ & $-0,554$ & 0,348 & 0,588 & 0,389 & $0,906^{*}$ & 0,356 & $-0,262$ & 0,293 & 0,550 & 0,560 & $-0,414$ & 0,399 \\
\hline
\end{tabular}

ES: error estándar.

* Clase Excelente como clase de referencia en el logística multinomial;

** $p<0,05$;

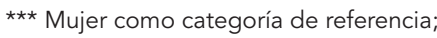

$\# \mathrm{p}<0,01$;

\#\# Enfermeras como categoría de referencia;

\#\#\# Atención primaria como categoría de referencia.

nos $16,22,39,40$. Sin embargo, y a tenor de los resultados, la formación parece darse en aquellos grupos de profesionales que tienen unos conocimientos óptimos en el uso de las técnicas, pero que tienen conocimientos deficitarios sobre aspectos conceptuales, materia vital para la prevención de la transmisión de microorganismos a través de las manos. Este área de mejora se da con una cierta mayor prevalencia entre el colectivo de otros profesionales de la salud y en centros de atención hospitalaria, información que favorece la elaboración de planes de formación más enfocados a colectivos profesionales específicos.

También conviene señalar, y siempre según las respuestas obtenidas de la encuesta, que el uso habitual de preparados de base alcohólica sucede con mayor probabilidad entre profesionales de la clase Déficit en transmisión, siendo además la clase más frecuente. Estos profesionales presentan unos conocimientos elevados sobre las técnicas de la higiene de las manos, pero bastante más escasos en conocimientos conceptuales para prevenir la transmisión. No hay que subestimar el posible sesgo de aquiescencia como explicación a tal hecho, por lo que debemos tomar con cautela dichas conclusiones.
Una vez sean corregidas dichas deficiencias de conocimientos, a través de la modificación de los planes formativos vigentes, y teniendo en cuenta que la clase Déficit en transmisión es el grupo más numeroso, podrían convertirse en profesionales de referencia para sus compañeros. Tanto ellos como aquellos que ya están demostrando sus conocimientos excelentes en higiene de manos podrían contribuir a catalizar y promover el cumplimiento de esta buena práctica. La importancia del liderazgo se contempla en varios estudios publicados recientemente sobre la higiene de las manos. En ellos se establece que deben tenerse en cuenta otras estrategias complementarias a la formación, uso de recordatorios, feedback a los profesionales y disponibilidad de productos, para mejorar la higiene de las manos. Entre estas estrategias destacan por su efectividad aquellas dirigidas al establecimiento de objetivos dentro del equipo y a la designación de líderes informales que fomenten la adherencia colectiva a la higiene de las manos 41,42 .

Entre las posibles limitaciones del presente estudio se contempla el carácter voluntario del cuestionario. El hecho de que la muestra no se haya obtenido a través de un muestreo probabi- 
lístico puede que haya afectado de algún modo al sesgo de respuesta a algunas preguntas. No obstante, el número elevado de encuestas recibidas y el presumible sesgo a favor de aquellos profesionales más motivados con la seguridad del paciente, los que han recibido formación, o utilizan de forma habitual preparados de base alcohólica, ha podido eventualmente ayudar a identificar cuáles son las lagunas más importantes en cuanto al conocimiento sobre la higiene de las manos y establecer así acciones de mejora prioritarias para llevar a la práctica.

Por último, es preciso subrayar que tras realizar una búsqueda exhaustiva en MEDLINE y Scopus no se han encontrado estudios que hayan utilizado el cuestionario de la OMS entre profesionales y con los cuales poder hacer una comparación de los resultados obtenidos, por lo que el presente trabajo se puede considerar un buen punto de partida para investigaciones futuras.

\section{Resumo}

O objetivo foi analisar o conhecimento sobre a higiene das mãos dos profissionais do Sistema de Saúde Andaluz (Espanha). Os inquéritos foram realizados em 2011, por meio do questionário Hand Hygiene Knowledge Questionnaire for Health-Care Workers, usando a técnica da análise de classe latente. Questões com porcentagens mais baixas de acertos são as relacionadas com a prevenção da transmissão de micro-organismos após exposição ao paciente por higiene das mãos e aquelas relacionadas com a utilização de preparações à base de álcool. Obtivemos um modelo de sete classes latentes. Principais diferenças sociodemográficas: os profissionais de conhecimentos mais baixos são homens, jovens e profissionais não relacionados à saúde; ter recebido treinamento anterior e não discriminar as pessoas com grande conhecimento. Concluímos que é necessária a revisão dos programas de formação de higiene das mãos para melhorar o conhecimento sobre aspectos conceituais da transmissão de micro-organismos pelas mãos.

Higiene das Mãos; Infecção Hospitalar; Segurança do Paciente; Conhecimentos, Atitudes e Prática em Saúde

\section{Colaboradores}

P. Pérez-Pérez, A. Bueno-Cavanillas y M. S. Alonso-Humada colaboraron activamente en la revisión bibliográfica, el comentario de los resultados, conclusiones y discusión. M. Herrera-Usagre participó activamente en la revisión bibliográfica, la implementación de las técnicas estadísticas, el comentario de los resultados, conclusiones y discusión. B. Buiza-Camacho colaboró activamente en la redacción de la introducción, la implementación de las técnicas estadísticas, el comentario de los resultados, conclusiones y discusión. M. Vázquez-Vázquez participó activamente en la redacción de la introducción, el comentario de los resultados, conclusiones y discusión.

\section{Agradecimientos}

A Vicente Santana López, Habibullah Rodríguez Contreras, Manuel Pacheco Mera, Inmaculada Rodríguez López, Bruno Pereira da Silva, Víctor Reyes Alcázar y Drew Linzer por sus sugerencias y ayuda técnica. A los revisores por sus valiosas contribuciones. A los responsables de los centros y unidades que trabajan fomentando la mejora en la higiene de las manos por su contribución a hacer posible este trabajo. 


\section{Referencias}

1. World Health Organization. Report on the burden of endemic health care-associated infection worldwide. Geneva: World Health Organization 2011.

2. Allegranzi B, Bagheri Nejad S, Combescure C, Graafmans W, Attar H, et al. Burden of endemic health-care-associated infection in developing countries: systematic review and meta-analysis. Lancet 2011; 377:228-41.

3. European Centre for Disease Prevention and Control. Annual epidemiological report on communicable diseases in Europe 2008: report on the state of communicable diseases in the EU and EEA/ EFTA countries. Stockholm: European Centre for Disease Prevention and Control; 2008.

4. Sociedad Española de Medicina Preventiva, Salud Pública e Higiene. EPINE 2011: Estudio de prevalencia de las infecciones nosocomiales en España. 220 Estudio. http://hws.vhebron.net/epine/Des cargas/EPINE\%202011\%20ESPA\%C3\%91A\%20Re sumen.pdf (accedido el 18/Ago/2014).

5. Aranaz-Andrés JM, Aibar C, Limón R, Mira JJ, Vitaller J, Agra Y, et al. A study of the prevalence of adverse events in primary healthcare in Spain. Eur J Public Health 2012; 22:921-5.

6. World Health Organization. WHO guidelines on hand hygiene in health care (advanced draft): a summary. Geneva: World Health Organization; 2005.

7. Pi-Sunyer Cañellas T, Banqué Navarro M, Freixas Sala N, Barcenilla Gaite F. Higiene de las manos: evidencia científica y sentido común. Med Clin (Barc) 2008; 131 Suppl 3:56-9.

8. Sánchez J, Rodríguez P. Podemos y debemos mejorar la higiene de manos. Tú decides. Enferm Infecc Microbiol Clín 2011; 29:1-3.

9. World Health Organization. WHO guidelines on hand hygiene in health care: first global patient safety challenge: clean care is safer care. Geneva: World Health Organization; 2009.

10. World Health Organization. Hand hygiene technical reference manual: to be used by health-care workers, trainers and observers of hand hygiene practices. Geneva: World Health Organization; 2009.

11. World Health Organization. Save lives: clean your hands. Guide to implementation a guide to the implementation of the WHO Multimodal Hand Hygiene Improvement Strategy. Geneva: World Health Organization; 2009.

12. World Health Organization. Save lives; clean your hands. Hand hygiene in outpatient and homebased care and long-term care facilities: a guide to the application of the WHO Multimodal Hand Hygiene Improvement Strategy and the "my five moments for hand hygiene" approach. Geneva: World Health Organization; 2012.

13. Burnett E. Perceptions, attitudes, and behavior towards patient hand hygiene. Am J Infect Control 2009; 37:638-42.

14. McLaughlin AC, Walsh F. Individual differences in judgments of hand hygiene risk by health care workers. Am J Infect Control 2011; 39:456-63.
15. Parmeggiani C, Abbate R, Marinelli P, Angelillo IF. Healthcare workers and health care-associated infections: knowledge, attitudes, and behavior in emergency departments in Italy. BMC Infect Dis $2010 ; 10: 35$

16. Sánchez-Payá J, Fuster-Pérez M, García-González C, Gracia-Rodríguez RM, García-Shimizu P, San Juan-Quíles A, et al. Evaluation of a program for updating recommendations about hand hygiene. An Sist Sanit Navar $2007 ; 30: 343-52$

17. Alp E, Ozturk A, Guven M, Celik I, Doganay M, Voss A. Importance of structured training programs and good role models in hand hygiene in developing countries. J Infect Public Health 2011; 4:80-90.

18. Sax H, Allegranzi B, Chraïti M-N, Boyce J, Larson E, Pittet D. The World Health Organization hand hygiene observation method. Am J Infect Control 2009; 37:827-34.

19. Kelcíkova S, Skodova Z, Straka S. Effectiveness of hand hygiene education in a basic nursing school curricula. Public Health Nurs 2012; 29:152-9.

20. Dierssen-Sotos T, Cal-López M, Navarro-Córdoba M, Rebollo-Rodrigo H, Antolín-Juarez FM, Llorca J. Factores asociados a la técnica correcta en la higiene de manos. Med Clin (Barc) 2010; 135:592-5.

21. Burnett E. Perceptions, attitudes, and behavior towards patient hand hygiene. Am J Infect Control 2009; 37:638-42.

22. Creedon SA. Healthcare workers' hand decontamination practices: compliance with recommended guidelines. J Adv Nurs 2005; 51:208-16.

23. Ferrer C, Almirante B. Higiene de manos: una prioridad para la seguridad de los pacientes hospitalizados. Enferm Infecc Microbiol Clin 2007; 25: 365-8.

24. Naikoba S, Hayward A. The effectiveness of interventions aimed at increasing handwashing in healthcare workers: a systematic review. J Hosp Infect 2001; 47:173-80.

25. Huis A, van Achterberg T, de Bruin M, Grol R, Schoonhoven L, Hulscher M. A systematic review of hand hygiene improvement strategies: a behavioural approach. Implement Sci 2012; 7:92.

26. Pittet D, Simon A, Hugonnet S, Pessoa-Silva CL, Sauvan V, Perneger TV. Hand hygiene among physicians: performance, beliefs, and perceptions. Ann Intern Med 2004; 141:1-8.

27. Hand Hygiene New Zealand Ringa Horoia Aotearoa. Guidelines on hand hygiene for New Zealand hospitals. Auckland: Hand Hygiene New Zealand Ringa Horoia Aotearoa; 2009.

28. Zimakoff J, Kjelsberg AB, Larsen SO, Holstein B. A multicenter questionnaire investigation of attitudes toward hand hygiene, assessed by the staff in fifteen hospitals in Denmark and Norway. Am J Infect Control 1992; 20:58-64.

29. Escuela Andaluza de Salud Pública, Servicio Andaluz de Salud, Consejería de Salud de la Junta de Andalucía. Resultados y calidad del Sistema Sanitario Público de Andalucía. Granada: Escuela Andaluza de Salud Pública/Sevilla: Servicio Andaluz de Salud; 2012. (Report GR 1229-2012). 
30. Observatorio para la Seguridad del Paciente, Agencia de Calidad Sanitaria de Andalucía. Cuestionario de conocimientos sobre la higiene de las manos para profesionales sanitarios. http://www. juntadeandalucia.es/agenciadecalidadsanitaria/ observatorioseguridadpaciente/gestor/sites/Por talObservatorio/higienedemanos/encuestas.html (accedido el 18/Ago/2014).

31. Escuela Andaluza de Salud Pública, Servicio Andaluz de Salud, Consejería de Salud de la Junta de Andalucía. Organización de la atención sanitaria pública en Andalucía. http://www.calidadsalu dandalucia.es/es/anexo_2.html (accedido el 28/ May/2013).

32. Ministerio de Sanidad. Cuestionario acerca de los conocimientos sobre la higiene de las manos destinado a los profesionales sanitarios. Madrid: Ministerio de Sanidad; 2009.

33. Hagenaars JA, McCutcheon AL. Applied latent class analysis. Cambridge: Cambridge University Press; 2002

34. Vermunt JK, Magidson J. Latent Class Analysis [Internet]. Tillburg: Statistical Innovations; 2006

35. Linzer DA, Lewis JB. poLCA: an R package for polytomous variable latent class analysis. J Stat Softw 2011; 42:1-29.

36. Amin T, Al Wehedy A. Healthcare providers knowledge of standard precautions at the primary healthcare level in Saudi Arabia. Healthcare Infection 2009; 14:65-72.

37. Bissett L. Interpretation of terms used to describe handwashing activities. Br J Nurs 2003; 12:536-42.
38. Martín-Madrazo C, Salinero-Fort MA, Cañada-Dorado A, Carrillo-De Santa-Pau E, Soto-Díaz S, Abánades-Herranz JC. Evaluación del cumplimiento de higiene de las manos en un área de atención primaria de Madrid. Enferm Infecc Microbiol Clin 2011; 29:32-5.

39. Dierssen-Sotos T, Robles-García M, Valbuena-Moya S. Lavado de manos: experiencias de dos comunidades autónomas. Med Clin (Barc) 2008; 131 Suppl 3:60-3.

40. Pittet D, Hugonnet S, Harbarth S, Mourouga P, Sauvan V, Touveneau S, et al. Effectiveness of a hospital-wide programme to improve compliance with hand hygiene. Infection Control Programme. Lancet 2000; 356:1307-12.

41. Huis A, Hulscher M, Adang E, Grol R, van Achterberg T, Schoonhoven L. Cost-effectiveness of a team and leaders-directed strategy to improve nurses' adherence to hand hygiene guidelines: a cluster randomised trial. Int J Nurs Stud 2013; 50:518-26.

42. Huis A, Schoonhoven L, Grol R, Donders R, Hulscher M, van Achterberg T. Impact of a team and leaders-directed strategy to improve nurses' adherence to hand hygiene guidelines: A cluster randomised trial. Int J Nurs Stud 2013; 50:464-74.

Recibido el 28/May/2013

Versión final presentada el 27/Jun/2014

Aprobado el 30/Jul/2014 